\title{
Revisión de las experiencias de revegetación con fines de restauración en bosques de la Argentina
}

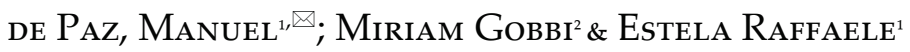 \\ ${ }^{1}$ Laboratorio Ecotono, INIBIOMA (Universidad Nacional del Comahue-CONICET). San Carlos de Bariloche, Argentina. \\ ${ }^{2}$ Departamento de Biología, CRUB - Universidad Nacional del Comahue e INIBIOMA.
}

\begin{abstract}
RESUMEN. La restauración de ambientes degradados se está implementando de manera creciente a nivel mundial con el fin de mitigar amenazas y recuperar los ecosistemas naturales. Los bosques de la Argentina no son ajenos a esta situación. Por ello, la Ley de Bosques Nativos promueve la protección, la recuperación y el uso sustentable de estos sistemas naturales. Sin embargo, en el país no existen revisiones de las experiencias que indiquen las técnicas más adecuadas para restaurar los bosques. Nos planteamos relevar el estado actual de las intervenciones de revegetación con fines de restauración y las técnicas más usadas en los distintos bosques de las ecorregiones de la Argentina. Para cada tipo de ecorregión evaluamos el efecto de las intervenciones más empleadas sobre la supervivencia de los plantines. La información la obtuvimos de revisión bibliográfica y encuestas a participantes de experiencias de restauración provenientes de los ámbitos científico, gubernamental y de la sociedad civil. Registramos escasa información publicada ( $50 \%$ de las experiencias). La mayoría de las superficies intervenidas fueron pequeñas y un porcentaje menor (43.1\%) fue monitoreado. Analizamos las técnicas de intervención más frecuentes para cada tipo de bosque y encontramos que usar riego y clausuras contra herbívoros genera un efecto positivo sobre la supervivencia de los plantines. Por el contrario, emplear enmiendas produjo un efecto negativo sobre la supervivencia. El control de la erosión y el uso de especies nodrizas varió según el tipo de ecosistema boscoso. La metodología de las encuestas fue apropiada para visibilizar las numerosas experiencias inéditas (50.4\%) que se realizaron en los diferentes bosques del país. El estudio incluye información variada provista por los actores locales, lo cual lo convierte en un insumo para futuros programas de restauración que incorporen la planificación territorial y la implementación de sistemas de evaluación y monitoreo.
\end{abstract}

[Palabras clave: ecorregiones, plantación, especies nativas, supervivencia de plantines]

Aвstract. Review of revegetation experiences for restoration purposes in the forests of Argentina. The restoration of degraded environments is being increasingly implemented worldwide in order to mitigate threats and recover natural ecosystems. Therefore, the forests of Argentina are not the exception to this situation. For this reason, the Native Forest Law promotes the protection, recovery and sustainable use of the native forests. However, there are no reviews in our country of past experiences that indicate which were the most appropriate techniques for restoring forests. Our aim was to survey the actual state of the revegetation for restoration purposes and interventions techniques most used in the different forest ecoregions of Argentina. We evaluated for each type of ecoregion the effect of the interventions most used on the survival of seedlings. The information was obtained from scientific publications and surveys to participants from restoration experiences of scientific fields, government and civil society. Little published information was recorded (50\% of the experiences). Most of the restored areas are small and a minor percentage $(43.1 \%)$ was monitored. We analyzed the most frequent intervention techniques for each type of forest and found that the survival of the seedlings was positive when using exclosures against herbivores and irrigation. In contrast, the use of amendments produced a negative effect on the survival seedlings; and erosion control and nurse species effects varied according to the type of forest ecosystem. Survey method was relevant to identify the significant number of unpublished experiences $(50.4 \%)$ carried out in the different regions of the country. The study includes a variety of information provided by local actors, which makes it an input for future restoration programs that incorporate territorial planning and the implementation of evaluation and monitoring systems.

[Palabras clave: ecoregions, plantation, native species, seedling survival]

Editora asociada: Ana Cingolani 凶depazm@comahue-conicet.gob.ar
Recibido: 11 de Diciembre de 2017

Aceptado: 16 de Enero de 2019 


\section{INTRODUCCIÓN}

En respuesta a los altos grados de deterioro y destrucción de ambientes naturales, la recuperación de ambientes degradados cobra cada vez mayor importancia a nivel mundial (González-Espinosa etal.2008). La restauración ecológica es una actividad deliberada que inicia o acelera la recuperación de un ecosistema degradado, para lo que considera su composición específica y la estructura de la comunidad, procesos funcionales y la resiliencia a la perturbación (SER 2004). Esta disciplina empieza a formalizarse en la década del ochenta (e.g., Society for Ecological Restoration International, SER 2004) y en los años '90 desarrolla un crecimiento exponencial de publicaciones científicas (Young et al. 2005) que continúa hasta el presente. En América Latina, actualmente la restauración está impulsada por los acuerdos internacionales (e.g., Convention on Biological Diversity, Bonn Challenge, Iniciativa 20x20, entre otros), con resultados diversos según las políticas asociadas a la restauración de cada país (Meli et al. 2017). Existen análisis globales sobre el avance de la restauración ecológica en el mundo (Young et al. 2005; Meli et al. 2016, 2017; Rey Benayas et al. 2017) y en la Argentina (Rovere 2015; Zuleta et al. 2015). En este país existen leyes que incluyen o se relacionan con la restauración (e.g., la Ley Nacional de Presupuestos Mínimos para la Protección Ambiental de Bosques Nativos N²6331- "Ley de bosques"- 2007; Zuleta et al. 2015). Sin embargo, el Plan Nacional de Restauración aún es incipiente (Meli et al. 2016). Por ello, es relevante revisar las experiencias de restauración llevadas a cabo en el país que sirvan para caracterizar su estado actual y contribuyan al desarrollo del mencionado plan.

Si bien existen diversos tipos de prácticas que intentan recuperar áreas degradadas, una de las más utilizadas y efectivas, tanto a nivel mundial como nacional, es la revegetación con especies nativas con fines de restauración ecológica (de aquí en más, revegetación). Esta práctica consiste en incorporar individuos de una o más especies vegetales (sembrar o plantar) en dichos sitios (Meli et al. 2017). También es una herramienta de restauración activa que se usa mucho en numerosos y recientes planes de manejo de bosque nativo con fines de conservación y recuperación en la Argentina (Peri and Ormaechea 2013; APN 2010; SAyDS 2014).
Existe información proveniente de distintos sectores (científico, técnico, particulares) que podría resultar relevante; específicamente, el conocimiento de los particulares, que no fue tenido en cuenta en revisiones previas y que los ambientes científico-técnico y de gestión desconocen (Meli et al. 2016). Consideramos como particulares a las personas involucradas en actividades de revegetación desde su compromiso ambiental, en muchos casos con participación en ONG. En este trabajo recopilamos y analizamos la información disponible (publicada y no publicada) sobre los ensayos de revegetación en bosques de la Argentina, para representar el estado actual de la actividad. Evaluamos, el efecto de las técnicas de revegetación más usadas en el país sobre la supervivencia de los plantines, un indicador sensible a corto plazo, medido en muchas prácticas de revegetación (en particular, en las que se realizaron monitoreos). Esta revisión se enfocó en los bosques de las distintas ecorregiones porque en las últimas décadas sufrieron uno de los procesos de deforestación más fuertes de su historia, según informa el Ministerio de Ambiente y Desarrollo Sustentable 2016 (MAyDS 2016).

\section{Materiales y Métodos}

\section{Áreas de estudio}

Consideramos los bosques de las distintas ecorregiones de la Argentina, que son el Bosque Andino-Patagónico, el Chaco Seco, el Chaco Húmedo, el Espinal, la Selva Misionera y las Yungas (Burkart et al. 1999). De estas ecorregiones, sólo una, los Bosques AndinoPatagónicos, corresponden a clima templado, mientras que las demás son subtropicales (Cabrera 1994). A continuación, mencionamos las características más relevantes de cada ecorregión (Burkart et al. 1999; MAyDS 2016).

Los Bosques Andino-Patagónicos abarcan una franja estrecha al oeste de la Patagonia, que abarca de $\operatorname{los} 35^{\circ}$ a los $55^{\circ} \mathrm{S}$. Tienen un gradiente de temperaturas N-S marcado (8$10^{\circ}$ hasta $6-5^{\circ}$, respectivamente) y un fuerte gradiente de precipitaciones (desde 500 a 3500 $\mathrm{mm} / \mathrm{año}$ ) que se expresa tanto N-S como E-O; éste último es consecuencia de la presencia de la Cordillera de los Andes. En general, los suelos son de origen volcánico, jóvenes y fértiles. El tipo fisonómico dominante es el bosque templado húmedo, que varía en especies con la altitud, la exposición de las 
laderas y la latitud. Hacia las zonas más xéricas del E, los parches de bosques y de arbustos se encuentran en una matriz de estepa. Diversas especies de Nothofagus dominan en toda su extensión. Las principales causas de degradación ambiental están asociadas a los incendios, la ganadería y la introducción de fauna y flora exóticas en el bosque.

El Chaco Seco abarca la mitad occidental de las provincias de Formosa y Chaco, una parte menor de Santa Fe, una porción oriental de Salta, Tucumán, Catamarca y La Rioja, casi la totalidad de Santiago del Estero y el norte de Santa Fe, Córdoba y San Luis. Es una planicie amplia de suave pendiente hacia el E, con formaciones serranas al sur. Existen dos gradientes climáticos generales en la ecorregión. Por un lado, las precipitaciones, asociadas al verano, que varían entre 500 y 800 mmanualesydisminuyenmarcadamentehacia el sudoeste. Por otro lado, la termoclimática, tropical, subtropical y templada de norte a sur. En particular, en la zona serrana, las precipitaciones y la temperatura presentan variaciones relacionadas tanto con un marcado gradiente altitudinal (altura máxima $~ 2880$ m s. n. m.) como con la exposición de las laderas. Los suelos presentan un gradiente de fertilidad de norte a sur; predominan los arenosos y con bajo contenido de materia orgánica. Especies de los géneros Schinopsis, Aspidosperma, Bulnesia y Prosopis, entre otros, son típicas de esta ecorregión. Los desmontes para la agricultura, la tala y la sobrecarga animal, junto con la invasión de especies exóticas e incendios, son las causas principales de degradación, al menos en las sierras.

La ecorregión del Chaco Húmedo ocupa el centro-noreste del país. Tiene lluvias intensas (1900 - 1050 mm/año) y la temperatura media anual varía entre 20 y $24{ }^{\circ} \mathrm{C}$. En el monte dominan especies de los géneros Schinopsis, Aspidosperma, Caesalpinia, Prosopis, Ziziphus y Geoffroea; en los palmares, Copernicia alba, y en las selvas en galería, árboles de los géneros Enterolobium, Tabebuia, Patagonula, Gleditsia, Ocotea y Phytolocca. Soporta una intervención antrópica considerable, con ganadería extensiva y una explotación maderera elevada. La expansión de cultivos agrícolas de alta tecnología agravó la situación ambiental.

La ecorregión del Espinal rodea la ecorregión del Pastizal Pampeano. Es una llanura plana a suavemente ondulada, con sabanas, pastizales y bosques bajos que, en gran parte, fueron convertidos a la agricultura y en la que se desarrollan diversas especies del género
Prosopis. El clima es cálido y húmedo en la zona norte; hacia el oeste y sur es templado y seco, con notables déficits hídricos. Los suelos de la parte norte son arcillosos e imperfectamente drenados; en el resto de la ecorregión son pobremente desarrollados, de textura gruesa y con poca materia orgánica. En la parte sur hay zonas medanosas. Se distinguen tres sectores, el mesopotámico, de clima húmedo con presencia de ñandubay (Prosopis affinis); el central, que es subhúmedo y representa una transición entre la Pampa y el Chaco, con presencia de algarrobos (Prosopis alba), y el del sur, semiárido, con presencia de caldén (Prosopis caldenia). La agricultura y la ganadería redujeron considerablemente la superficie de los bosques de esta ecorregión. La salinización y la compactación de los suelos son consecuencias relevantes de estas actividades productivas.

La Selva Misionera ocupa casi toda la provincia de Misiones. Presenta valores muy altos de biodiversidad y se desarrolla hasta los 30 ó $40 \mathrm{~m}$ en 4 ó 5 estratos verticales. Posee clima cálido y húmedo, con lluvias relativamente bien distribuidas en el año, que varían desde $2000 \mathrm{~mm}$ al NE hasta 1800 al S. Son típicos los suelos rojos, producto de la oxidación de rocas volcánicas con alto porcentaje de hierro y aluminio, que varían desde profundos y arcillosos (mesetas) hasta poco evolucionados y poco profundos (en relieve escarpado). Entre las especies arbóreas más importantes de la ecorregión, se encuentra Araucaria angustifolia, aunque con distribución restringida en la actualidad, y especies del género Cedrela, palmito (Euterpre edulis) y palo rosa (Dalbergia nigra), entre muchas otras. Las principales causas de degradación se asocian con la deforestación, la agricultura migratoria que genera bosques fragmentados con síntomas de degradación y con la expansión de la frontera agropecuaria.

En la Argentina, las Yungas están vinculadas con la Cordillera de los Andes de manera discontinua desde Salta hasta Catamarca. La altitud varía entre 400 y 3000 m s. n. m.; las precipitaciones, entre 900 y $1300 \mathrm{~mm} /$ año. Esta diversidad en condiciones ambientales se asocia con valores de biodiversidad también muy altos y tipos forestales heterogéneos en distintos pisos altitudinales, desde Selvas Pedemontanas cálidas, con presencia de palo blanco (Calycophyllum multiflorum), palo amarillo (Phyllostylon rhamnoides), tipa (Tipuana tipu) y pacará (Enterolobium contortisiliquum), Selva Montana templado- 
cálido con maroma (Ficus maroma), los laureles (Cinnamomum porphyrium, Nectandra pichurim y Ocotea puberula) y pocoy (Inga spp.), hasta Bosque Montano templado con heladas invernales frecuentes y presencia de pino del cerro (Podocarpus parlatorei), yoruma colorada (Roupala meisneri), aliso del cerro (Alnus acuminata) y el nogal criollo (Juglans australis). Esta ecorregión ocupa el segundo lugar entre las de mayor degradación (Primer Inventario Nacional de Bosques Nativos) y las principales causas son la pérdida del hábitat y la fragmentación de los ecosistemas.

Armamos una base de datos unificada de proyectos de revegetación en la Argentina a partir de una búsqueda bibliográfica y de encuestas semi-estructuradas. Como

Tabla 1. Descripción/estados posibles de las variables de clasificación de los proyectos de revegetación con especies nativas en bosques en la Argentina.

Table 1. Description/possible states of classification variables of native species revegetation projects in Argentina.

Variable Descripción/estados

Tipo de bosque intervenido según regiones Bosque Andino-Patagónico, Yungas, Selva Misionera, Espinal, Chaco Seco biogeográficas (Burkart et al. 1999) y Chaco Húmedo.

Provincia/s

Localización de la/s intervención/es

Fuente/s de información

Publicaciones científicas, trabajos en reuniones científicas, encuestas, informes técnicos, planes de manejo y libros

Año de inicio del proyecto

Inicio formal o informal de las actividades de gabinete o campo del proyecto

Fuente/s de financiamiento

Estado, organizaciones intermedias, empresas, particulares, internacional (gubernamentales y no gubernamentales, academias de ciencias, organismos internacionales, etc.)

Tipo de instituciones participantes del proyecto

Superficie intervenida

Objetivo/s generales

Objetivo/s particulares

Disturbios y usos previos de la tierra

Universidad, organismos o empresas del Estado nacional, provincial o municipal (Sec. Medioambiente, bosques, YPF, CORFONE), Instituto de investigación, INTA, APN, particulares, escuelas, comunidad de pueblos originarios, ONG

Superficie en ha intervenida a lo largo del desarrollo de cada experiencia

Restauración de un área degradada o investigación o educación ambiental

Recuperación de un área degradada, educación ambiental, incremento de la productividad, mejorar el uso turístico o control de especies exóticas

Factores de degradación previos del sitio intervenido: fuego, tala, ganado, invasión y plantación de especies exóticas, minería

Tipo de intervención/es

Plantación o siembra de especies nativas, clausuras contra animales, plantación bajo especies nodrizas, control de especies exóticas, uso de enmiendas (e.g., compost, mulch, hidrogel), raleos, riego, distintos tiempos de plantación y de tipos de plantín, procedencia de semillas y de plantines, control de erosión, traslado de suelos, protección contra insectos

Forma de vida plantada/sembrada

Especie/s plantada/sembrada

Árbol, arbustos, hierbas y trepadoras

Especies incorporadas en el sitio intervenido

Año en los que se incorporó individuos a campo

Total de individuos de especies nativas incorporados al sitio intervenido

En el caso de semillas la edad es 0 , en el caso de haber distintas edades se registró cada una independientemente

Número de individuos por cada uno de los tipos de intervención y por especie

Tiempo trascurrido desde la plantación hasta el o los monitoreos de supervivencia

Porcentaje de individuos vivos sobre el total de plantados al año de la plantación

Porcentaje de individuos vivos sobre el total de plantados del último monitoreo registrado 
fuentes bibliográficas consideramos trabajos académicos relevados en Google Académico y Scopus. Utilizamos como filtros las palabras restauración ecológica, bosque y Argentina en trabajos publicados hasta fines de 2015 . Relevamos 552 publicaciones de artículos y de reuniones científicas, libros y artículos de divulgación. Estas publicaciones se sometieron a un segundo filtro considerando las palabras clave revegetación, enriquecimiento, plantación o siembra de especies nativas, o Bosque Andino-Patagónico, Yunga, Selva Misionera, Espinal, Chaco Seco o Chaco Húmedo (Ch-H), o ley de bosques o nombres de especies emblemáticas en ensayos de restauración. Simultáneamente, contactamos a 430 especialistas, gestores y técnicos con incumbencias en proyectos de restauración o temáticas relacionadas, a los que les solicitamos que respondieran una encuesta sobre experiencias (intervenciones) de revegetación.

A partir de estos filtros y de la lectura de los trabajos seleccionados y de las encuestas construimos una base de datos de 137 proyectos (Anexo 1) y 20 variables que representan las características más relevantes de los distintos tratamientos de revegetación utilizados, intervenciones (Tabla 1). Cada proyecto contaba con un número variable de experiencias de revegetación (entre 1 y 17). Las encuestas se utilizaron para completar la información de cada proyecto.

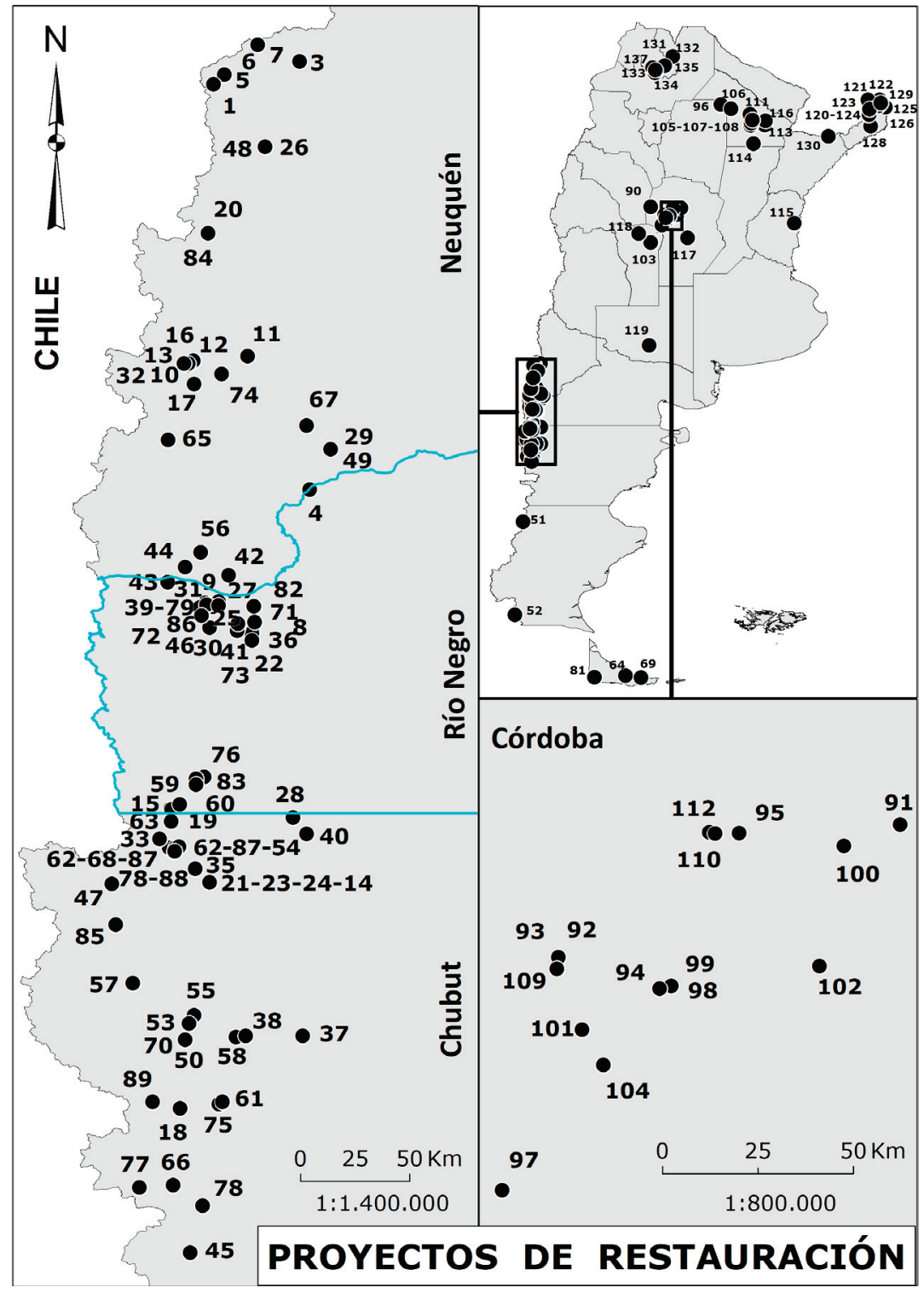

Figura 1. Proyectos de restauración de bosques recopilados en esta revisión. Cada punto referencia un proyecto (ver Anexo 1).

Figure 1. Forest restoration projects compiled in this review. Each point references a project (see Annex 1). 


\section{Análisis de datos}

Para evaluar las tendencias temporales en el desarrollo de estudios de revegetación en los sistemas boscosos del país analizamos la cantidad de proyectos en función del año de inicio de los ensayos de restauración. Para determinar la existencia de una respuesta de tipo umbral en el número acumulado de ensayos de revegetación realizamos un análisis de regresión segmentada (Toms et al. 2003). Este tipo de análisis identifica los posibles cambios en la pendiente del modelo de regresión mediante la estimación de los puntos umbrales que estiman el punto de inflexión del número acumulado de experiencias de revegetación. Seleccionamos el mejor modelo (uno o dos umbrales y regresión lineal) mediante el criterio de información de Akaike (AIC) y el mínimo delta-AIC ( $\triangle$ AIC $>2$ ) para identificar diferencias significativas entre modelos (Burnham and Anderson 2002).

Para cada provincia del país, estimamos la superficie total intervenida en relación al área de bosque total. También determinamos los años de monitoreo promedio por área fitogeográfica a partir de los datos informados en cada experiencia.

El efecto de las intervenciones más utilizadas sobre la supervivencia de plantines al primer año luego de la plantación fue abordado por regiones, para lo que se implementó un estudio de meta-análisis log-response ratio $(\mathrm{Rr})$ (Hedges et al. 1999). Este estudio sólo se pudo aplicar para aquellos sitios intervenidos con actividades de revegetación que contaban con información de un sitio control, es decir de muy similares características, pero sin intervención. Si en un mismo proyecto existían plantaciones en distintos años, sitios geográficos o tipos de intervención, consideramos a cada una de ellas como una experiencia distinta a los fines del meta-análisis. En este estudio, calculamos el response ratio como $\ln \left(\mathrm{Xt}_{\mathrm{t}} / \mathrm{Xc}_{\mathrm{c}}\right)$, donde $\mathrm{Xt}$ es la supervivencia media (variable respuesta) en los distintos tipos de intervenciones y Xc es la supervivencia media en los lugares donde no hubo intervenciones (control).

Este meta-análisis establece la magnitud del efecto de cada tipo de intervención y su desvío. $\mathrm{Si} \mathrm{Rr}=0$ ó si los intervalos de confianza

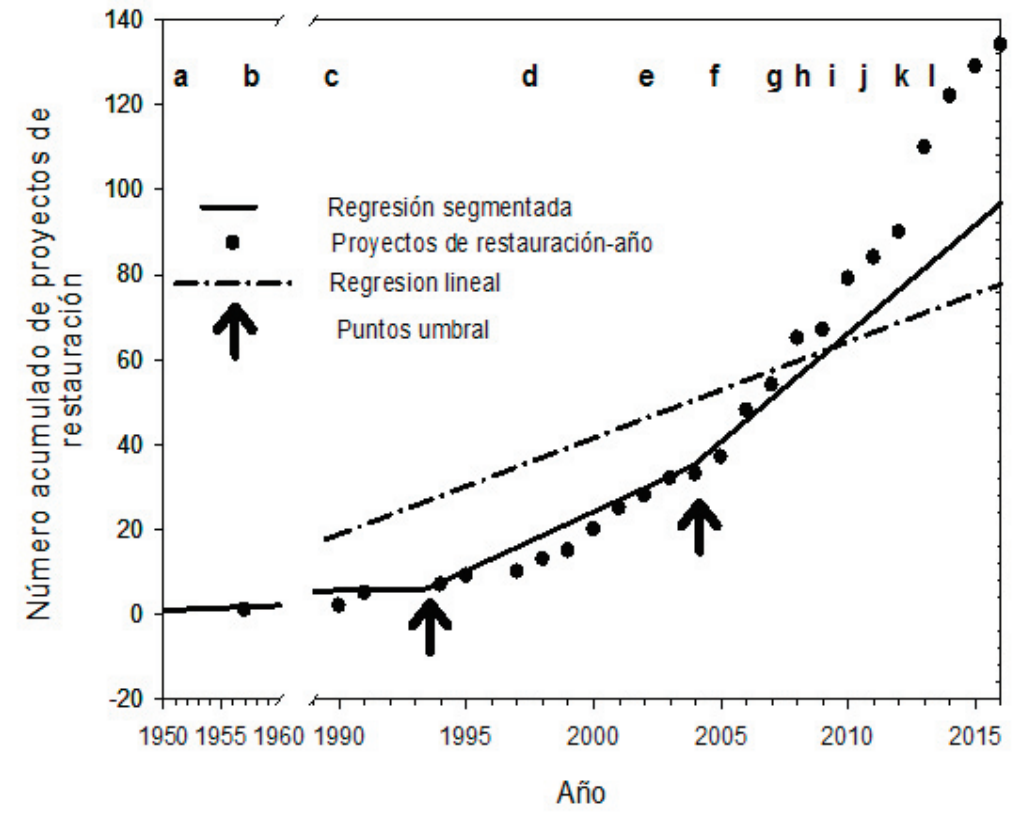

Figura 2. Regresión segmentada (Tabla 2) y regresión lineal (Anexo 2 de proyectos acumulados relacionados a restauración de la Argentina por año. Las letras indican hechos destacados relacionados con la conservación y restauración de bosques: a) 1948-Ley Nacional No 13273 de Defensa Forestal, b) Primera experiencia registrada por esta recopilación, c) 1989-Ley provincial N 2342 de Prevención de Degradación Ambiental, d y f-1997-2005 Primer inventario de bosques nativos, e-2002-Ley Nacional General del Ambiente $\mathrm{N}^{\circ}$ 25675, g-2007-Ley nacional No 26331 de Presupuestos Mínimos de Protección Ambiental de los Bosques Nativos, h-1-2008-2014-Leyes provinciales de Presupuestos Mínimos de Protección Ambiental de los Bosques Nativos

Figure 2. Piece-wise regression (Table 2) and linear regression (Annex 3) of accumulated number of projects related to restoration of Argentina per year. The letters indicate outstanding facts related to the conservation and restoration of forests: A) 1948-National Forest Law $\mathrm{n}^{\circ} 13273$, b-First experience of this compilation, c-1989-Provincial Law $\mathrm{N}^{\circ} 2342$ of Prevention of Environmental Degradation, d and f-1997-2005-First native forests inventory, e-2002-National General Environmental Law No 25675, g-2007-National Law No 26331 of Minimum Environmental Protection Budgets of Native Forests, h-1-2008-2014-Provincial Laws of Minimum Budgets of Environmental Protection of Native Forests 
de $\mathrm{Rr}$ solapan el 0, indica que el efecto de las intervenciones sobre la supervivencia de los plantines no es significativo. Cuando $\mathrm{Rr}$ es positivo, indica que la supervivencia es significativamente mayor con las intervenciones. Por el contrario, si el Rr es negativo, la supervivencia es mayor en los controles. El efecto del uso de riego, de especies nodrizas y de clausuras contra grandes herbívoros sobre la supervivencia de las especies plantadas/sembradas sólo fue analizado en Bosque Andino-Patagónico y Chaco Seco debido a la poca información para otros tipos de bosque. Por el mismo motivo, el efecto del uso de enmiendas y del control de especies exóticas se analizó sólo en el Bosque Andino-Patagónico y el control de erosión en Bosque Andino-Patagónico, Chaco Seco, Selva Misionera y Yungas.

\section{Resultados}

De los 137 proyectos que integran la base de datos, 89 (65\%) corresponden a los Bosques Andino-Patagónicos, 23 (16.8\%) al Chaco Seco, $11(8 \%)$ a la Selva Misionera, 7 (5.1\%) a las Yungas, 4 (2.9\%) al Chaco Húmedo y 3 al Espinal (2.2\%).

La mayoría de los organismos contactados poseían limitada y poco sistematizada información sobre la actividad, como se detalla en los resultados subsiguientes. El 49.6\% de los proyectos recopilados provienen de fuentes de información publicadas, mientras que la información de los restantes proviene de las comunicaciones efectuadas en las encuestas (Anexos 1 y 2).

Las experiencias de revegetación recopiladas en este trabajo, que abarcan el período 1957-2015, muestran un incremento de la actividad en las últimas dos décadas (91.4\% de las experiencias) (Figura 2) y una respuesta umbral con dos puntos de inflexión (años

Tabla 2. Regresión segmentada de proyectos de revegetación con especies nativas por año $\left(r^{2}=0.99\right)$.

Table 2. Piece-wise regression of native species revegetation projects per year $\left(r^{2}=0.99\right)$.

\begin{tabular}{cccccc}
\hline & GL & CM & F & $P$ & \\
\hline Regresión & 5.24 & 6440.8 & 1276.4 & $<0.01$ & \\
& Coeficiente & & $t$ & $P$ & VIF \\
Umbral-1 & $1993.5 \pm 0.8$ & & 2500.6 & $<0.01$ & 2.78 \\
Umbral-2 & $2003.9 \pm 0.4$ & & 4744.1 & $<0.01$ & 8.80 \\
\hline
\end{tabular}

Este modelo cumplió supuestos de normalidad (Kolmogoroff), homogeneidad de varianza y autocorrelación (Durbin-Watson)
1993 y 2004, Figura 2 y Tabla 2, AIC=-33.2). El número acumulado de experiencias en función del tiempo (Figura 2) muestra tres etapas: 1) inicio de la actividad en el país, con experiencias aisladas (1957-1993), 2) crecimiento moderado de la actividad (19932004), y 3) crecimiento acelerado (2004-2015). Otras regresiones mostraron un ajuste menor (lineal) o no cumplieron con los supuestos del análisis (regresión segmentada-1 umbral) (Anexo 2).

\section{Caracterización de los ensayos de revegetación}

Del total de proyectos relevados, $29.2 \%$ tuvo como objetivo general sólo la recuperación del área degrada y 24.2\% únicamente hacía referencia a la investigación; mientras tanto, menos del $1 \%$ hacía referencia sólo a la educación en relación a la restauración. El resto de los proyectos combinaron dos o tres de los objetivos mencionados. Entre los objetivos específicos citados se destaca la recuperación del área degradada (61.3\%) y le siguen las combinaciones de recuperación con educación ambiental (16.1\%), con la productividad (7.3\%) y con el control de exóticas (6.6\%). Todos los demás objetivos, solos o combinados, tiene valores menores a $5 \%$.

El 64\% de los proyectos relevados informó la superficie intervenida, que en total representa 1490 ha. De esta superficie, el 65\% corresponde a objetivos mixtos de recuperación de áreas degradadas, investigación y educación ambiental, y el 3\% al objetivo exclusivo de investigación. A nivel regional, la mayor superficie intervenida fue en Chaco Seco, Bosque Andino-Patagónico y Selva (Figura 3a). A nivel de provincias, Córdoba contiene la mayor superficie intervenida (Figura 3b) y Chubut y Rio Negro las mayores superficies relativas a la cantidad de bosque de cada provincia (Figura 3c). La mediana de superficie por proyecto a nivel país es 2 ha ( 2 ha en Bosque Andino-Patagónico y Chaco Húmedo, 3 ha en Chaco Seco y 1 ha en Yungas y Selva Misionera).

La fuente de financiamiento más utilizada fue el Estado (83\%), seguido por ONG (12.1\%), privados $(9.6 \%)$ y, por último, los fondos internacionales (7\%). En su mayoría hubo una sola línea de financiamiento por proyecto, excepto en el Chaco Seco, donde $70 \%$ de los proyectos tuvo más de una fuente.

Los principales tipos de disturbio y usos de la tierra en los sitios, previo a la implementación 


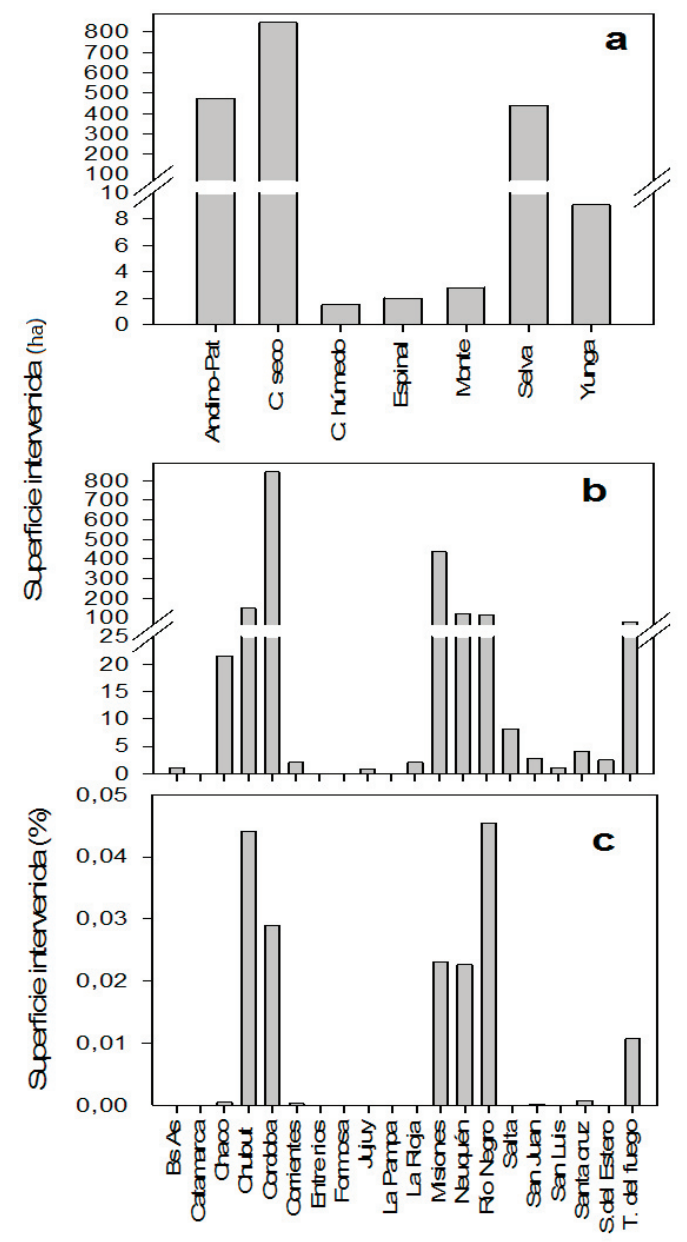

Figura 3. Superficie total intervenida por experiencias de revegetación con fines de restauración por tipo de bosque (a) y por provincia en ha (b) y relativa al total de superficie de bosque en cada provincia (c).

Figure 3. Total area covered by revegetation for restoration experiences by forest type (a) and by province in ha (b) and relative to total forest surface by province (c).

de proyectos de revegetación, ya sea solos o combinados con otros, fueron incendio (54.2\%, principalmente en Chaco Húmedo, Bosque Andino-Patagónico y Chaco Seco), ganado $(30.5 \%$, principalmente en Bosque Andino-Patagónico, Chaco Seco y Espinal) y tala $(11.9 \%$, principalmente en Yungas, Selva Misionera, Bosque Andino-Patagónico y Chaco Seco).

La mayoría de las experiencias de revegetación se realizaron a través de la plantación de especies nativas $(89.9 \%)$ y con clausuras contra herbívoros $(50 \%)$, aunque otras intervenciones, tales como el control de la erosión, el control de especies exóticas y los raleos también se usaron en algunos tipos de bosques (Figura 4). El control de especies exóticas fue planteado principalmente como una práctica para favorecer la instalación de las especies nativas (18\%) más que como un objetivo específico en sí mismo $(6 \%)$ y, en general, no fue monitoreado en el tiempo, salvo en monitoreos de supervivencia en los Bosque Andino-Patagónico (58.7 $\pm 5.5 \%$ ).

Los árboles fueron la forma de vida más empleada (Anexo 1) en las experiencias de revegetación $(85 \%$ del total de los proyectos y $99.6 \%$ de las plantas utilizadas). Las experiencias de revegetación se monitorearon en el $43.1 \%$ de los proyectos, y en pocos casos el seguimiento superó los 3 años. Un $72.5 \%$ de las experiencias de plantación monitoreadas tuvieron supervivencias mayores a $30 \%$ de los individuos plantados al primer año de la plantación; esta supervivencia fue similar a la del primer año en la última medición efectuada $\left(\chi^{2}=0.125, P<0.72\right)$.

Los principales aspectos mencionados en las encuestas como positivos para el desarrollo de las actividades (Anexo 3) fueron similares para los distintos tipos de bosque. Estos aspectos son el presupuesto apropiado, la edad y el estado sanitario de los plantines (en particular para la Selva Misionera y las Yungas). En cambio, los principales aspectos considerados como negativos variaron con el tipo de bosque (Anexo 3), y fueron la herbivoría (en Bosque Andino-Patagónico), la degradación del ambiente (en Yungas), la baja disponibilidad de plantines (en el Bosque Andino-Patagónico, en el Chaco Húmedo, en la Selva Misionera y en las Yungas) y la sequía (en las Yungas y en el Chaco Seco). De las encuestas también surge la poca información disponible de las especies que se desea reintroducir y de las características ecológicas de los sitios donde se realizan las intervenciones. La falta de financiamiento para el mantenimiento de los tratamientos es otro factor limitante o negativo que fue mencionado en las experiencias relevadas.

\section{Uso de técnicas de restauración}

Laintervención más utilizada en los proyectos relevados fue la plantación, con una frecuencia mayor a $90 \%$ en todos los bosques considerados (Figura 4). El tipo y la frecuencia de uso de las diferentes técnicas de restauración asociadas con las plantaciones variaron con la región. La supervivencia de plantines al primer año, como variable respuesta, cambió según la técnica implementada.

Clausura contra animales o herbívoros. Este tipo de intervención se implementó en 

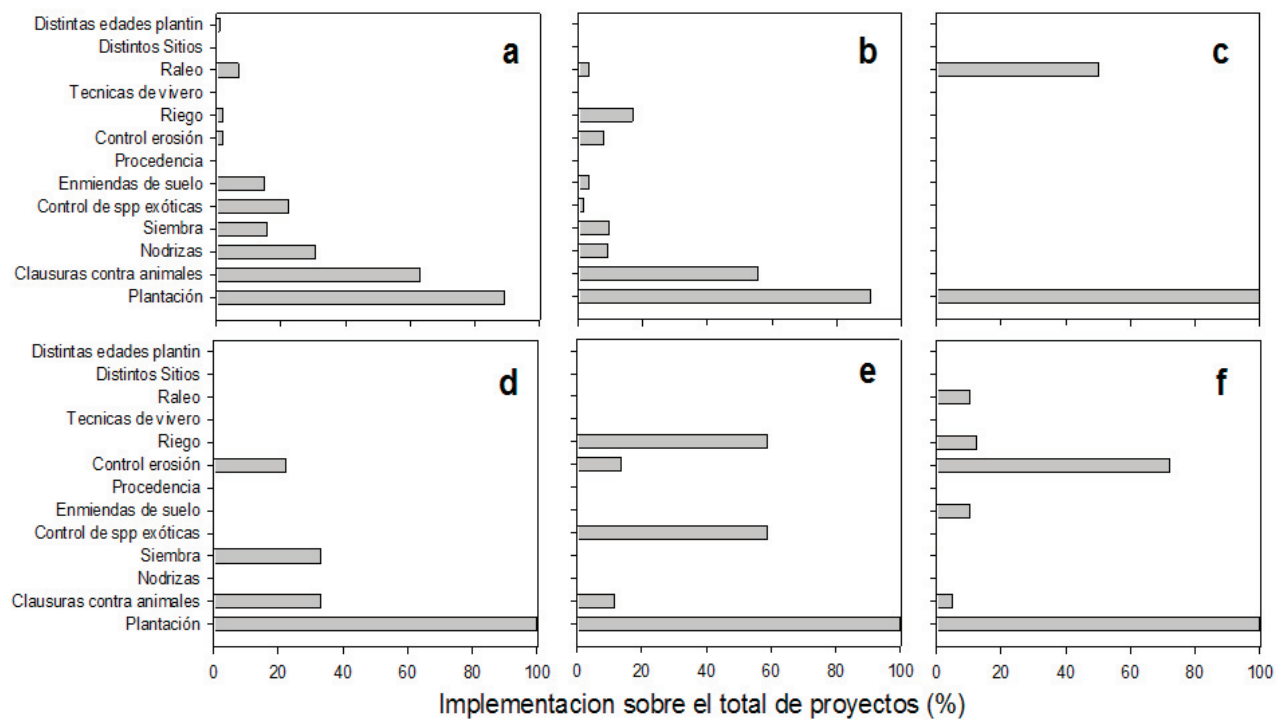

Figura 4. Técnicas utilizadas en las intervenciones realizadas en los proyectos de restauración recopilados y número de proyectos entre paréntesis según tipo de bosque: a) Bosque Andino-Patagónico (89), b) Chaco Seco (23), c) Chaco Húmedo (4), d) Espinal (3), e) Selva Misionera (11) y f) Yungas (7).

Figure 4. Techniques used in the interventions carried out in restoration projects collected and amount of project by forest type: a) Bosque Andino-Patagónico (89), b) Chaco Seco (23), c) Chaco Húmedo (4), d) Espinal (3), e) Selva Misionera (11) y f) Yungas (7).

la mayoría de los tipos de bosque, excepto en Chaco Húmedo, con diferentes frecuencias (Figura 4). Sólo se contó con datos apropiados para evaluar el efecto de las clausuras sobre la supervivencia de las plantas en Bosque Andino-Patagónico y Chaco Seco. En ambos casos, la aplicación de esta técnica aumentó la supervivencia de las plantas (5a).

Nodrizas. El uso de plantas leñosas con fines de protección de los individuos plantados (nodrizas) en proyectos de revegetación se reportó sólo en dos ecorregiones (Bosque Andino-Patagónico y Chaco Seco, Figura 4). En Bosque Andino-Patagónico tuvo una alta implementación (Figura 4a) y un efecto positivo sobre la supervivencia (Figura 5b). Contrariamente, en Chaco Seco, la implementación fue baja $(10.1 \%$ de las experiencias, Figura $4 \mathrm{~b}$ ) y el efecto sobre la supervivencia fue negativo (Figura $5 b$ ).

Controldeerosión. Estetipodeintervenciones se implementaron en todas las ecorregiones, salvo $\mathrm{CH}-\mathrm{H}$ (Figura 4), con una frecuencia de aplicación mayor en Yungas $(72.3 \%$ de los proyectos) (Figura 4f). Sólo pudimos evaluar los efectos sobre la supervivencia en Chaco Seco y en Bosque Andino-Patagónico, y fueron positivos (Figura $5 \mathrm{c}$ ).
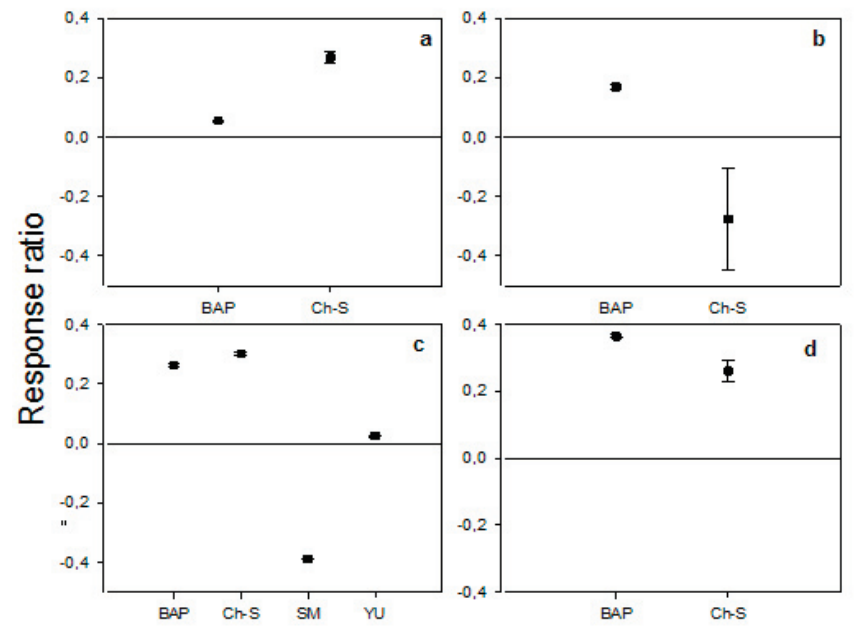

Figura 5. Índice response-ratio $(\mathrm{Rr}) \mathrm{de}$ la supervivencia al año de plantaciones de especies nativas en las siguientes técnicas de intervenciones: clausuras contra herbívoros (a), nodrizas (b), control de erosión (c) y riego (d) en distintas ecorregiones (Bosque AndinoPatagónico (BAP), Chaco Seco (Ch-S), Selva Misionera (SM) y Yungas (YU).

Figure 5. Response-ratio ratio (Rr) of annual survival of native species plantations considering the following intervention techniques: herbivore exclosures (a), nurse species (b) erosion control (c) and irrigation (d) for different ecoregions (Bosque AndinoPatagónico (BAP), Chaco Seco (Ch-S), Selva Misionera (SM) y Yungas (YU). 
Enmiendas. la aplicación de algún tipo de producto que mejore las características del suelo (e.g., compost, mulch, fertilizantes) fue reportado en pocos proyectos de Bosque Andino-Patagónico, Chaco Seco y Yungas, (15\% de las experiencias). Sólo pudimos analizar el efecto de las enmiendas en Bosque Andino-Patagónico, en donde se registró un efecto negativo sobre la supervivencia $(\mathrm{Rr}=-$ $0.12 \pm 0.01)$.

Riego. Esta técnica de intervención se aplicó en cuatro ecorregiones (Figura 4) con proporciones distintas que van desde 1.6\% en Bosque Andino-Patagónico hasta 57.8\% en Selva Misionera. Sólo se informaron datos de supervivencia de plantas con riego en Bosque Andino-Patagónico y Chaco Seco, e indicaron un efecto positivo (Figura 5d).

Control de exóticas. Este tipo de intervención se implementó, en orden de importancia, en Selva Misionera, Bosque Andino-Patagónico y Chaco Seco (Figura 4). No pudimos analizar el efecto sobre la supervivencia por falta de sitios control (con plantación de plantines de especies nativas sin erradicación de las especies exóticas).

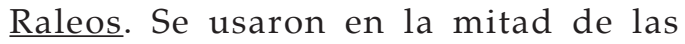
experiencias de Chaco Húmedo y en menos de $10 \%$ de las de Yungas, Bosque AndinoPatagónico y Chaco Seco (Figura 4). No se informaron resultados de supervivencia asociados.

Siembras. Se implementaron, en orden de importancia, en Espinal, Bosque AndinoPatagónico y Chaco Seco, con porcentajes menores al 35\%. No se informaron valores de supervivencia.

\section{DISCUSIÓN}

Este trabajo presenta la primera recopilación efectuada a partir de fuentes académicas y no académicas de la revegetación de bosques de la Argentina. Si bien existen análisis globales sobre el avance de la restauración en el país (Rovere 2015; Zuleta et al. 2015), la base de datos de este estudio contempla información no publicada perteneciente a organismos gubernamentales y no gubernamentales que no fue incluida en revisiones previas. La metodología empleada (encuestas) fue apropiada para visibilizar la gran cantidad de experiencias inéditas $(50.4 \%)$ realizadas en los diferentes bosques de las ecorregiones del país y que no están siendo consideradas para revisar o evaluar las intervenciones de restauración en los ecosistemas boscosos de la Argentina.

Actualmente, se considera que la restauración ecológica es la estrategia del futuro para conservar y rehabilitar los ecosistemas a largo plazo a nivel mundial. A partir de la década del 2000, el crecimiento de las experiencias de revegetación en la Argentina fue exponencial. La respuesta umbral del número de experiencias de revegetación se relaciona con el auge mundial de esta actividad que comienza en el año 1993, junto con el inicio de distintas iniciativas de investigación y agrotécnicas que impulsaron la actividad en el país. El crecimiento de la actividad a partir de 2004 se puede explicar por varios factores, entre ellos: a) respuesta a la demanda social por conservar y recuperar los bosques (Zuleta et al. 2015), b) mayor conocimiento científicotécnico a nivel nacional e internacional, c) estabilidad económica del país y mayor financiación hacia esta actividad (CepalStats 2016). Las condiciones socio-ambientales mencionadas más la consolidación de herramientas legales (Ley de Bosques nacional y provinciales equivalentes 2008-2014, Ley General del Ambiente 2002, Código Civil 2015; Zuleta et al. 2015) y la conformación de redes académico-técnicas locales (REA) y regionales (REDLAND 2005; REACRE 2007; SIACRE 2013; Zuleta 2015) también fueron impulsores de esta actividad.

Entre los proyectos de revegetación relevados, $75 \%$ incluyó entre sus objetivos la restauración de áreas degradadas y $62 \%$ la investigación. Los resultados obtenidos en función de la fecha de inicio de los proyectos muestran que es una actividad en pleno desarrollo. Las fuentes de financiamiento mencionadas muestran que está estimulada mayoritariamente por el Estado, en particular porque financia los ensayos de investigación en los que se prueban y mejoran las técnicas. Las iniciativas exclusivamente privadas (e.g., particulares, empresas u ONG con interés en conservación) son relativamente pocas y recientes.

Si bien el crecimiento de intervenciones aumentó mucho en las últimas décadas, los proyectos que superan las 3-4 ha son pocos (Figura2) y se encuentran aún en etapas experimentales. Los planes de restauración de la Ley de Bosques marcaron el crecimiento reciente de la actividad, en especial en tierras de particulares (85\%, SAyDS 2014). Al mismo 
tiempo que se desarrollan las diferentes técnicas de intervención se están definiendo las bases conceptuales de la restauración de dichos ecosistemas. En ese sentido, la recomendación de muchos investigadores es combinar estrategias pasivas y activas con el fin de incrementar la resiliencia, la productividad y la funcionalidad, para así aumentar la supervivencia de estos ecosistemas ante los cambios globales.

Los estudios a largo plazo o las parcelas permanentes, que incluyen remediciones en el tiempo, son una herramienta útil para analizar los resultados que caracterizan o describen un ecosistema, sus interacciones y servicios ecosistémicos, así como las respuestas del sistema a las intervenciones humanas con objetivos de recuperación. En este sentido, las observaciones a largo plazo en ensayos de restauración pueden desempeñar un papel clave para comprender las diferentes técnicas aplicadas (Herrick et al. 2006) y permitirán incorporar una evaluación objetiva del cumplimiento de las metas planteadas. Sin embargo, nuestros resultados muestran que se monitoreó menos del 50\% de las experiencias. Proyectos y líneas de financiamiento enfocados a la evaluación a largo plazo permitirán una mayor capitalización de las experiencias implementadas. A partir de estas experiencias monitoreadas en el tiempo fue posible apreciar que, en la mayoría de estos casos, cuando la supervivencia de la especie plantada al año fue mayor al $30 \%$, estos valores se repiten en años subsiguientes.

\section{Evaluación de las de técnicas de restauración}

La plantación comoestrategia de revegetación fue la más utilizada, lo cual coincide con revisiones anteriores (Meli et al. 2017). A su vez, usar clausuras para evitar el ramoneo fue una intervención positiva, muy aplicada en Bosque Andino-Patagónico y Chaco Seco. Otra técnica utilizada en experiencias de revegetación es usar enmiendas (e.g., compost (Basil et al. 2009) o mulch (Gobbi et al. 2015), aunque fue sólo pudo ser evaluada en el Bosque AndinoPatagónico. El efecto general de la aplicación de enmiendas fue negativo. Sin embargo, en otros países o ambientes esta técnica resultó efectiva para disminuir las condiciones de estrés que afectan la supervivencia de los plantines. Varios estudios indican los efectos positivos en la disponibilidad de nutrientes por aplicación de compost (e.g., Laos et al. 2000; Kowaljow and Mazzarino 2007). Entre los efectos de enmiendas como mulch se citan la reducción de la evaporación de agua del suelo (Unger and Parker 1976; Shanging and Unger 2001), de la erosión por lluvia y del escurrimiento superficial, el aumento de la permeabilidad del suelo y la atenuación de las temperaturas a la altura del cuello de la planta (Wiegand Davies 1999).

A nivel mundial, una técnica extendida en ambientes áridos y semiárido es usar especies nodrizas (e.g., Padilla and Pugnaire 1997; Ashton et al. 1997; Gómez-Aparicio et al. 2009). Esta técnica fue poco implementada en experiencias de revegetación en la Argentina, excepto en los Bosques Andino-Patagónicos y Chaco Seco. En los Bosques Andino Patagónicos, este tratamiento produjo un efecto positivo sobre la supervivencia de los plantines, en particular en sitios mésicos y xéricos y con alta radiación, y fue particularmente probada en Austrocedrus chilensis (Svriz et al. 2013; Urretavizcaya et al. 2013; Gobbi et al. 2015). En el Chaco Seco, este tipo de tratamiento fue exitoso para el establecimiento de Polylepis australis en cárcavas en terrazas con suelos poco profundos (Landi and Renison 2010). En este último trabajo, el efecto de los arbustos como plantas nodriza no resultó positivo en sitios con suelo profundo. Esto podría deberse a que en estos suelos hay un mayor contenido de arcillas que permiten una mayor retención de agua, lo cual produce un balance hídrico más favorable para $P$. australis.

La evaluación de las experiencias de revegetación para controlar las especies exóticas no fue posible debido a la ausencia de sitios control. Sin embargo, es interesante notar que en los Bosques Andino-Patagónicos se registró una supervivencia de plantines muy similar en sitios con erradicación de exóticas, en comparación con plantaciones en bosque nativo (60.2\% y $67.2 \%$, respectivamente). Esto impulsa la idea de la factibilidad de recuperar bosque nativo reemplazado por especies exóticas. Algunas experiencias en la Selva Misionera y en el Chaco Seco fueron informadas como exitosas, pero no obtuvimos resultados suficientes para realizar un análisis al respecto.

Las intervenciones para el control de la erosión fueron positivas en Bosque AndinoPatagónico, Chaco Seco y Yungas. Numerosos antecedentes de ambientes semiáridos y bosques templados coinciden con estos resultados. En esos ambientes, las mejoras en la retención de suelo a través de la combinación 
de obras de infraestructura con materiales del sitio, que estabilicen el suelo y detengan los procesos de erosión, consiguieron buenos resultados (e.g., Ludwig and Tongway 1996; Vallejo et al. 2006; Burylo et al. 2007). Estas estructuras retienen también materia orgánica, nutrientes y propágulos, los que promueve el establecimiento y la regeneración de plantas (Ludwig and Tongway 1996; Renison et al. 2010).

\section{Conclusiones}

Esta revisión de experiencias de revegetación muestra el estado actual de la restauración de los bosques de la Argentina, a la cual consideramos como un componente integral de la conservación de la biodiversidad conjuntamente con la preservación, uso sostenible y generación de conocimiento.

Asimismo, esta revisión ha permitido sistematizar y hacer accesible información que se encontraba dispersa, poco disponible y valiosa para la evaluación de las estrategias que han sido probadas para mejorar el éxito de la revegetación.

Actualmente, desarrollar proyectos de restauración ecológica es cada vez más frecuente, sin embargo, es importante conocer bien el funcionamiento de los ecosistemas en donde se desarrollen los proyectos. Este entendimiento resultará en la implementación de proyectos efectivos en términos ecológicos y económicos. Sumado a lo anterior, está la capacidad de identificar los cambios que está causando y que causará el cambio climático en ecosistemas sensibles a este fenómeno. Nosotros recomendamos identificar especies resistentes a los cambios ambientales asociados al cambio global para mantener y recuperar las funciones y servicios ecosistémicos.

Evaluar el éxito de los proyectos de restauración ecológica es fundamental para justificar el uso de la restauración en los ecosistemas y mejorar las prácticas que se utilizan. Actualmente existen extensas discusiones en torno a las variables que definen y miden las restauraciones exitosas, monitoreos o evaluación de proyectos. Es por esto que realizamos este estudio que revisa, a nivel regional (Argentina), las tendencias y el estado actual de la restauración e identifica los vacíos de información claves que necesitan ser abordados.

Nosotros consideramos a la sobrevivencia como un indicador o atributo de restauración que mide rápidamente el éxito de las intervenciones, además que es la medición más frecuente que se registra en las experiencias relevadas. Sin embargo, existen otros indicadores relevantes que han sido poco considerados y que están asociados a la revegetación con especies nativas, por ejemplo, la recolonización de la fauna y la recuperación de las condiciones de fertilidad del suelo en los sitios en recuperación. Somos conscientes que los estudios y trabajos futuros de restauración en la Argentina deben tener en cuenta otras mediciones, además de la sobrevivencia, que cuantifiquen los servicios ecosistémicos, por ejemplo, resultados socioeconómicos que puedan ayudar a entender los beneficios y costos de la restauración en el manejo de los recursos naturales. Higgs (1997) argumentó la necesidad de mirar más allá de la ecología e incluir aspectos históricos, sociales, culturales, políticos, y estéticos para definir los objetivos de restauración. Los debates sobre estos nuevos objetivos ya comenzaron en muchas partes del mundo, pero aún faltan en la Argentina. Centrarse únicamente en los resultados ecológicos de la restauración es insuficiente para evaluar el éxito de la restauración. Identificar y medir los beneficios socioeconómicos y los valores socioculturales vinculados con los sitios intervenidos que proporcionan los ecosistemas comienza a ser relevante en muchas partes del mundo.

Agradecimientos. Esta revisión no habría sido posible sin el aporte de todos los gestores, docentes, científicos, técnicos, empresas, ONG y particulares que colaboraron con información para la construcción de la base de datos. Agradecemos la colaboración y sugerencias de A. Pérez y S. Caracotche para la base de datos y encuestas, las sugerencias de Y. Sabatier para el mapa, los comentarios y críticas de los dos evaluadores anónimos y los aportes en la edición del manuscrito de L. Cavallero.

\section{REFERENCIAS}

APN. 2010. Programa de las Naciones Unidas para el desarrollo en Argentina: Restauración y control de factores de deterioro en los bosques nativos de los parques nacionales. APN, Buenos Aires.

Ashton, P. M. S., S. Gamage, I. A. U. N. Gunatilleke, and C. V. S. Gunatilleke. 1997. Restoration of a Sri Lankan rainforest: using Caribbean pine Pinus caribaeaas a nurse for establishing late-successional tree species. Journal of Applied Ecology 34:915-925. https://doi.org/10.2307/2405282. 
Basil, G., M. J. Mazzarino, L. Roselli, and F. Letourneau.2009. Efecto del compost de biosólidos en la producción de plantines de Austrocedrus chilensis (ciprés de la cordillera). Ciencias del suelo [online] 27:49-55

Burkart, R., N. Bárbaro, R. Sánchez, and D. Gómez. 1999. Eco-regiones de la Argentina. Administración de Parques Nacionales. Secretaria de Recursos Naturales y Desarrollo Sustentable. Presidencia de la Nación. Pp. 43 en M. Burylo, F. Rey and P. Delcros. 2007. Abiotic and biotic factors influencing the early stages of vegetation colonization in restored marly gullies (Southern Alps, France). Ecological Engineering 30:231-239. https://doi.org/10.1016/ j.ecoleng.2007.01.004.

Burnham, K. P., and D. R. Anderson. 2002. Model selection and multi model inference: a practical information-theoretic approach. Springer-Verlag, New York, USA

CepalStat. 2016. Bases de Datos y publicaciones estadísticas de la Comisión Económica para América Latina y el Caribe (CEPAL). URL: interwp.cepal.org/cepalstat/WEB_cepalstat.

Gobbi, M. E., K. Heinemann, M. de Paz, C. Núñez, and R. Herrero. 2015. Revegetación con especies arbóreas nativas en áreas ecotonales del NO de Patagonia: herramientas para la creación de islas de regeneración. Pp. 62-65 en M. A. Marcó and C. I. Lavallol (eds.). Investigación Forestal 2011-2016. Los Proyectos de Investigación Aplicada. MinAgri., UCAR. $1^{\mathrm{a}}$ ed. CABA.

Gómez Aparicio, L. 2009. The role of plant interactions in the restoration of degraded ecosystems: a meta analysis across life forms and ecosystems. Journal of Ecology 97:1202-1214. https://doi.org/10.1111/j.1365-2745.2009.01573.x.

González-Espinosa, M., J. M. Rey-Benayas, and N. Ramírez-Marcial. 2008. Restauración de bosques en América Latina. Mundi-Prensa. Madrid.

Hedges, L. V., and I. Olkin 1985. Statistical Methods for Meta-Analysis. Academic Press, INC, Orlando.

Herrick, J. E., G. E. Schuman, and A. Rango. 2006. Monitoring ecological processes for restoration projects. Journal for Nature Conservation 14:161-171. https://doi.org/10.1016/j.jnc.2006.05.001.

Higgs, E. S. 1997. What is good ecological restoration? Conservation Biology 11:338-348. https://doi.org/10.1046/ j.1523-1739.1997.95311.x.

Kowaljow, E., and M. J. Mazzarino. 2007. Soil restoration in semiarid Patagonia: Chemical and biological response to different compost quality. Soil Biology and Biochemistry 39:1580-1588. https://doi.org/10.1016/ j.soilbio.2007.01.008.

Landi, M. A., and D. Renison. 2010. Forestación con Polylepis australis en suelos erosionados de las Sierras Grandes de Córdoba: evaluación del uso de terrazas y vegetación nodriza. Ecología Austral 20:47-55

Laos, F., P. Satti, I. Walter, M. J. Mazzarino, and S. Moyano. 2000. Nutrient availability of composted and noncomposted residues in a Patagonian Xeric Mollisol. Biology and Fertility of Soils 31:462-469. https://doi.org/10.1007/ s003740000192.

Ludwig, J. A., and D. J. Tongway. 1996. Rehabilitation of semiarid landscapes in Australia. II. Restoring vegetation patches. Restoration Ecology 4:398-406. https:// doi.org/10.1111/j.1526-100X.1996.tb00192.x.

Ministerio de Ambiente y Desarrollo Sustentable. 2016. Informe del estado de ambiente 2016. Buenos Aires. Pp. 454.

Meli, P., F. F. Herrera, F. Melo, S. Pinto, N. Aguirre, K. Musálem, C. Minaverry, W. Ramírez, and P. H. S. Brancalion. 2016. Four approaches to guide ecological restoration in Latin America. Restoration Ecology 25:156-163. https:// doi.org/10.1111/rec.12473.

Meli, P., K. D. Holl, J. M. Rey Benayas, H. P. Jones, P. C. Jones, D. Montoya, and D. Moreno. 2017. A global review of past land-use, climate, and active vs. passive restoration effects on forest recovery. PLOSOne 12:e0171368. https: //doi.org/10.1371/journal.pone.0171368.

MAyDS. 2016. Informe del Estado del Ambiente 2016. Min. De Ambiente y desarrollo sustentable de Argentina, Buenos Aires, Argentina.

Padilla, F. M., and F. I. Pugnaire. 2006. The role of nurse plants in the restoration of degraded environments. Frontiers in Ecology and the Environment 4:196-202. https://doi.org/10.1890/1540-9295(2006)004[0196:TRONPI]2.0.CO;2.

Peri, P., and S. Ormaechea. 2013. Relevamiento de los bosques de ñire (Nothofagus antarctica) en Santa Cruz: base para su conservación y manejo. INTA, Buenos Aires, Argentina.

Rey-Benayas, J. M. R., P. Barral, and P. Meli. 2017. Lecciones de cuatro meta-análisis globales sobre la restauración de la biodiversidad y los servicios ecosistémicos. Ecología Austral 27:193-198. https://doi.org/10.25260/ EA.17.27.1.1.252.

Renison, D., I. Hensen. R. Suárez, A. A. M. Cingolani, P. Marcora, and M. A. Giorgis. 2010. Soil conservation in Polylepis mountain forests of Central Argentina: is livestock reducing our natural capital? Austral Ecology 35:435-443. https: //doi.org/10.1111/j.1442-9993.2009.02055.x.

Rovere, A. E. 2015. Review of the science and practice of restoration in Argentina: increasing awareness of the discipline. Restoration Ecology 23:508-512. https://doi.org/10.1111/rec.12240.

SAyDSN. 2014. Ley N ${ }^{\circ} 26331$ de presupuestos mínimos de protección ambiental de Bosques nativos. Informe de Estado de implementación 2010-2014. SAyDSN, Buenos Aires, Argentina. Pp. 29.

SER (Society for Ecological Restoration). 2004. The SER International Primer on ecological restoration. URL: www.ser.org/docs/default-documentlibrary/english.pdf (último acceso: 15/10/2017).

Shanging, J., and P. W. Unger. 2001. Soil water accumulation under different precipitation, potential evaporation and straw mulch conditions. Soil Science Society of America Proceedings 65:442-448. https://doi.org/10.2136/ sssaj2001.652442x.

Svriz, M., M. A. Damascos, H. Zimmermann, and I. Hensen. 2013. The exotic shrub Rosa rubiginosa as a nurse 
plant. Implications for the restoration of disturbed temperate forests in Patagonia, Argentina. Forest Ecology and Management 289:234-242. https:/ / doi.org/10.1016/j.foreco.2012.09.037.

Toms, J. D., and M. L. Lesperance. 2003. Piecewise regression: a tool for identifying ecological thresholds. Ecology 84(8):2034-2041. https://doi.org/10.1890/02-0472.

Unger, P. W., and J. J. Parker. 1976. Evaporation reduction from soil with wheat, sorghum, and cotton residues. Soil Science Society of America Proceedings 40:938-942. https:/ / doi.org/10.2136/sssaj1976.03615995004000060035x.

Urretavizcaya, M. F., and G. E. Defossé. 2013. Effects of nurse shrubs and tree shelters on survival and growth of two Austrocedrus chilensis seedling types in a forest restoration trial in semiarid Patagonia, Argentina. Annals of Forest Science 70:21-30. https://doi.org/10.1007/s13595-012-0234-z.

Vallejo, R., J. Aronson, J. G. Pausas, and J. Cortina. 2006. Restoration of Mediterranean woodlands. Pp. 193-207 in J. van Andel and J. Aronson. Restoration Ecology: The New Frontier. Blackwell Publ., Malden, USA.

Wiegand Davies, H. M. 1999. Efecto de la utilización de mulch de acícula de pino, corteza de pino, paja con guano de caballo y guano de pavo sobre la producción del palto (Persea americana Mill) cv. Hass. Universidad Católica de Valparaíso, Quillota.

Wortley, L., J. Hero, and M. Howes. 2013. Evaluating Ecological Restoration Success: A Review of the Literature. Restoration Ecology 21:537-543. https://doi.org/10.1111/rec.12028.

Young, T. P., D. A. Petersen, and J. J. Clary.2005. The ecology of restoration: historical links, emerging issues and unexplored realms. Ecology Letters 8:662-673. https://doi.org/10.1111/j.1461-0248.2005.00764.x.

Zuleta, G., A. E. Rovere, D. Pérez, P. I. Campanello, B. Guida Johnson, C. Escartín, and J. Aronson. 2015. Establishing the ecological restoration network in Argentina: from Rio1992 to SIACRE 2015. Restoration Ecology 23:95-103. https: //doi.org/10.1111/rec.12198. 